\title{
Neumoencéfalo intraventricular a tensión secundario a cirugía transesfenoidal: presentación de un caso y revisión de la literatura
}

\author{
F. Ruiz-Juretschke; O. Mateo-Sierra; B. Iza-Vallejo y R. Carrillo-Yagüe
}

Servicio de Neurocirugía. Hospital General Universitario Gregorio Marañón. Madrid.

\section{Resumen}

El neumoencéfalo a tensión (NT) es una complicación rara de los abordajes transesfenoidales. Se describe el caso de una mujer de 37 años intervenida de un adenoma de hipófisis por vía transesfenoidal que presentó rinolicuorrea autolimitada en el postoperatorio. A los tres días la paciente desarrolló un deterioro progresivo de conciencia, amnesia anterógrada y cefalea, detectándose en la TC un NT intraventricular. Se realizó un tratamiento urgente con drenaje ventricular externo bilateral y taponamiento nasal anterior con buena respuesta clínica. De modo diferido se procedió a la revisión del abordaje esfenoidal lográndose el cierre definitivo del defecto dural sin recidiva posterior.

El NT después de la cirugía por vía esfenoidal suele ocurrir tras la aparición de una fístula de LCR debido al sellado incompleto del seno esfenoidal. La colocación de un drenaje lumbar postoperatorio parece predisponer a esta complicación. El enfoque terapéutico combinado del NT mediante drenaje ventricular externo y reparación del seno esfenoidal ofrece un resultado óptimo con una solución rápida para el deterioro neurológico del paciente y evita las recidivas.

PALABRAS CLAVE: Fístula de líquido cefalorraquídeo. Drenaje lumbar. Adenoma de hipófisis. Neumoencéfalo. Cirugía transesfenoidal.

Intraventricular tensión pneumocephalus after transsphenoidal surgery: a case report and literature review

\section{Summary}

Tension pneumocephalus is a rare complication of transsphenoidal approaches. The case of a 37 year old woman with a transsphenoidal resection of a pituitary adenoma who presented self-limited rhinoliquorrhea postoperatively is reported. Three days later the

Recibido: 7-05-06. Aceptado. 7-07-06. patient developed progressive decreased consciousness, amnesia and headache, showing an intraventricular tension pneumocephalus on CT scan. Urgent treatment with bilateral external ventricular drainage and anterior nasal tamponade was performed with good clinical outcome. Later transsphenoidal sealing of the dural defect was achieved without recurrence.

Tension pneumocephalus following transsphenoidal surgery usually occurs after the presentation of a cerebrospinal fluid leak due to an incomplete sealing of the sphenoid sinus. The postoperative insertion of a lumbar drainage seems to be a predisposing condition for this complication. The combined approach of tension pneumocephalus with external ventricular drainage and repair of the sphenoid sinus offers optimal results solving the acute neurological deterioration and avoiding recurrence.

PALABRAS CLAVE: Cerebrospinal fluid fistula. Lumbar drainage. Pituitary adenoma. Pneumocephalus. Transphenoidal surgery.

\section{Introducción}

La incidencia de neumoencéfalo a tensión (NT) tras cirugía transesfenoidal para la resección de adenomas hipofisarios es extremadamente baja, con 4 casos descritos por Sawka et al. en más de 2500 pacientes intervenidos en la Clínica Mayo entre 1976 y $1998^{9}$. Aunque el neumoencéfalo es una complicación frecuente en pacientes con fractura craneal y tras cirugías craneales generalmente cursa de forma asintomática y se reabsorbe de manera espontánea. El neumoencéfalo a tensión se define como el acúmulo patológico de aire intracraneal asociado a un deterioro neurológico por hipertensión intracraneal o efecto masa. Su localización suele ser subdural, aunque se ha descrito a nivel subaracnoideo, intracerebral e intraventricular. Presentamos un caso clínico de neumoencéfalo intraventricular sintomático secundario a una cirugía transesfenoidal que ilustra la dificultad diagnóstica y terapéutica de este tipo de complicación contrastándolo con la literatura actual. 


\section{Caso clínico}

Una mujer de 37 años sin antecedentes de interés consultó por pérdida de visión y fotopsias, asociadas a astenia y déficit de atención. La exploración neurológica confirmó una disminución de la agudeza visual en ambos ojos sin defecto campimétrico. La resonancia magnética mostró una lesión selar con extensión supraselar y compresión del quiasma. El estudio hormonal fue normal. La paciente fue intervenida mediante abordaje transesfenoidal. Se incidió la dura en X observándose una mínima salida de LCR que cedió con la aplicación de Surgicel®. La lesión, con aspecto macroscópico de adenoma, se resecó por completo. Finalmente la apertura dural se selló con pegamento de fibrina Tissucol ${ }^{\circledR}$ y se colocó un drenaje lumbar en quirófano para prevenir la fístula de LCR. El diagnóstico patológico fue de adenoma de hipófisis no funcionante.

La evolución postoperatoria inicial fue buena, excepto por una rinolicuorrea discreta que cedió al bajar el nivel del drenaje lumbar, lo cual provocó una clínica transitoria de hipotensión de líquido. La tomografía computarizada (TC) de control no mostró indicios de complicación (Fig. 1). A los tres días de retirar el drenaje lumbar se instauró un cuadro progresivo de cefalea, somnolencia, amnesia anterógrada, edema facial llamativo y disminución del nivel de conciencia. En la TC se detectó aire intraventricular con niveles hidroaéreos en las astas frontales y el tercer ventrículo con dilatación de los mismos, así como aire en la región selar y el espacio subaracnoideo asociado a una disminución de los surcos de la convexidad (Fig 2). Se intervino de urgencia mediante dos trépanos frontales bajo anestesia local y se introdujo un catéter de drenaje ventricular en cada asta frontal, observándose salida de aire a presión y LCR claro. Además se realizó un taponamiento nasal anterior bilateral. El estudio del LCR fue normal. La paciente mejoró llamativamente de nivel de conciencia y el resto de los síntomas remitió de forma paulatina. Posteriormente se realizó un taponamiento y sellado por vía nasal transesfenoidal de la silla turca y del seno esfenoidal con grasa y pegamento de fibrina, colocando un nuevo drenaje lumbar. En la TC de control se observó menor cantidad de aire en los ventrículos con resolución de la dilatación de los mismos (Fig 3). Al alta hospitalaria la paciente se encontraba asintomática.

\section{Discusión}

El NT es una complicación poco frecuente de la cirugía transesfenoidal y representa un importante reto diagnóstico y terapéutico. En este trabajo se revisan brevemente los aspectos más relevantes de su fisiopatología y tratamiento. Según el momento de aparición el NT se clasifica como: precoz, si se presenta en los primeros siete días, por la

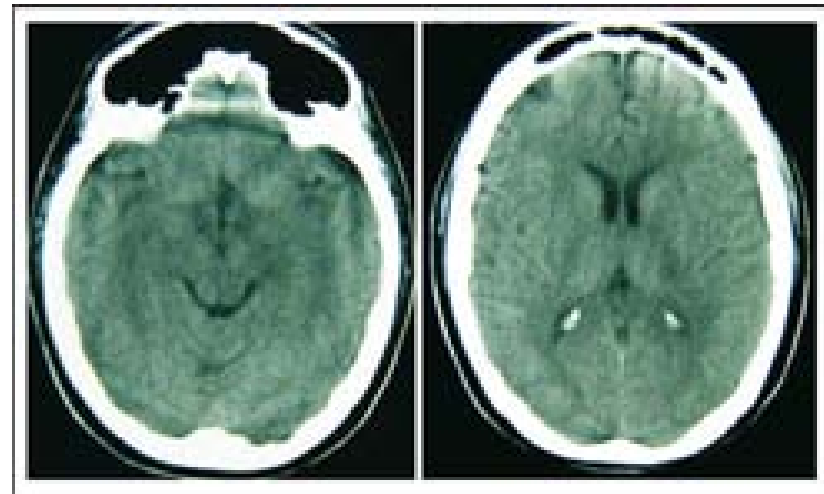

Figura 1. TC craneal a las 48 horas de la intervención transesfenoidal sin que se objetive aire intracraneal.

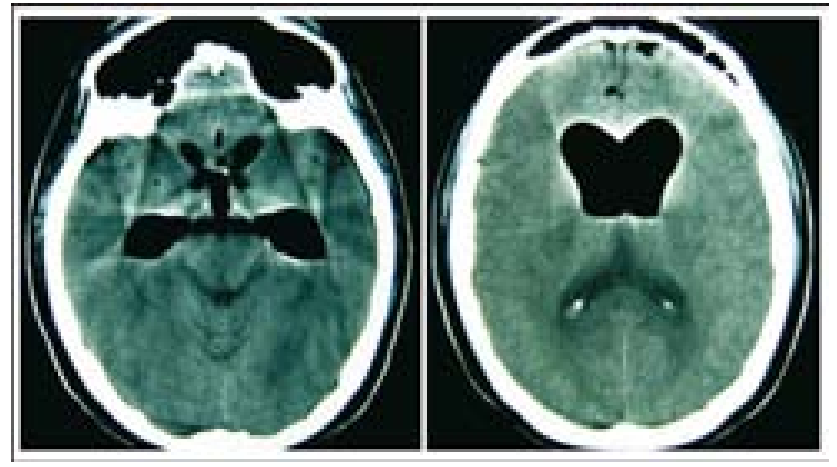

Figura 2. TC de control a los 8 días de la intervención coincidiendo con el deterioro clínico de la paciente en el que se aprecia aire a tensión en el tercer ventrículo y ventrículos laterales con niveles hidroaéreos en ambas astas frontales. También se aprecia aire a nivel subdural paraselar, frontal y en la cisura interhemisférica.

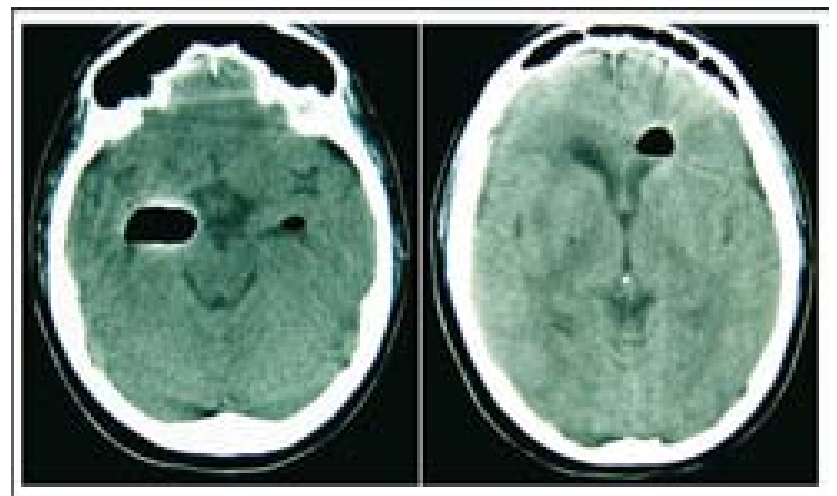

Figura 3. TC craneal a las 24 horas de drenar el neumoencéfalo, en el que se ven restos de aire intraventricular sin apenas signos de tensión.

entrada masiva de aire debido a la posición del paciente en quirófano, o tardío, si se instaura después de una semana ${ }^{10}$. El desarrollo de un NT tardío suele estar condicionado por la presencia de una fístula de LCR que se produce en 1$10 \%$ de las intervenciones transesfenoidales ${ }^{6}$. La entrada 
de aire se explica según tres modelos teóricos complementarios $^{12}$ : (a) el modelo de presión negativa: existe un gradiente de presión que favorece la entrada de aire; (b) el modelo de la botella de gaseosa invertida: la salida continua de LCR favorece la entrada de aire que asciende en forma de burbujas reemplazado al líquido; (c) el modelo de válvula unidireccional: la forma de la fístula y su relación con el cerebro permite la entrada de aire pero impide su salida. Sólo es posible el neumoencéfalo cuando la presión intracraneal es menor a la atmosférica. Por eso en condiciones normales tiende a salir líquido por la fístula hasta que las presiones se igualan. En ese momento los factores que incrementen la salida de líquido (la maniobra de Valsalva y los cambios posturales), aquéllos que reducen la presión intracraneal (el drenaje lumbar, la deshidratación, la reducción de masa tumoral por radioterapia ${ }^{4}$ ) y los que incrementan la presión del aire extradural (la apnea obstructiva del sueño ${ }^{9}$ y la ventilación a presión positiva) generan un gradiente de presión que favorece la entrada de aire. De todos éstos, la colocación de un drenaje lumbar para el tratamiento conservador de la fístula de LCR es el factor que más predispone al desarrollo de neumoencéfalo. En un estudio reciente de 16 pacientes con drenaje lumbar por rinolicuorrea, 3 desarrollaron un neumoencéfalo no tensional, aunque sólo en uno se objetivó sobredrenaje de líquido ${ }^{1}$.

En el caso de nuestra paciente existió una fístula de LCR con rinolicuorrea inicial en la que el tamaño del defecto dural impedía que se generase un efecto valvular y por tanto un neumoencéfalo. Es frecuente que en el momento de constatarse la presencia de aire intracraneal a tensión el paciente ya no tenga una rinolicuorrea evidente ${ }^{2,7}$. Posteriormente tras un tratamiento conservador con el drenaje lumbar se consiguió frenar la salida de líquido, aunque el defecto dural aún era permeable al aire. Esto posibilitó la formación del NT favorecido por la persistencia del drenaje lumbar durante tres días más.

En este caso el aire no sólo se acumuló en el espacio subdural y subaracnoideo de la base del cráneo y la convexidad, sino que penetró en el sistema ventricular provocando una dilatación de las astas frontales que fue la causante de la clínica de deterioro del nivel de conciencia con confusión y amnesia. Esta es la forma de presentación más frecuente de esta complicación que no tiene ninguna sintomatología específica que oriente al diagnóstico ${ }^{10}$. Además se localizó aire en los tejidos blandos de la cara provocando un edema facial llamativo. No se apreció focalidad neurológica ni signos meníngeos como en otros $\operatorname{casos}^{3,5}$. Aunque el LCR fue normal es posible observar pleocitosis en esta situación ${ }^{11}$.

En cuanto al tratamiento se han descrito diversas estrategias, desde un enfoque conservador, esperando la resolución espontánea del cuadro por reabsorción del aire a lo largo de varios meses ${ }^{13}$, hasta el drenaje del aire intracraneal atrapado o la reparación quirúrgica de la fístula de LCR. En general, se recomienda prevenir la fístula de LCR y sus complicaciones realizando en todos los casos un taponamiento de la silla turca y del seno esfenoidal con grasa, fascia u otros materiales, independientemente de que haya habido fuga de LCR durante la intervención ${ }^{4}$. En el caso de desarrollar un neumoencéfalo como consecuencia de una fístula de LCR postoperatoria, debe de retirarse el drenaje lumbar empleado para el tratamiento conservador de la fístula inmediatamente, porque favorece la entrada de más aire ${ }^{7}$. El tratamiento conservador ha demostrado ser insuficiente en la mayoría de los $\operatorname{casos}^{4,8}$. La mayoría de los autores recomiendan la reparación de la silla turca y el taponamiento del seno esfenoidal por vía transnasal como tratamiento de elección ${ }^{2,4,5,10}$.

En nuestro caso el deterioro neurológico de la paciente hizo imperativa la evacuación del aire introduciendo dos catéteres intraventriculares en ambas astas frontales. Esto permitió revertir la dilatación ventricular normalizándose la clínica. Posteriormente se decidió revisar la zona de la fístula dural por vía transesfenoidal para evitar que volviese a penetrar aire. Para ello se taponaron la silla turca y el seno esfenoidal con grasa sellándolos con pegamento de fibrina. Haran et al ${ }^{4}$ recomiendan retrasar el drenaje del aire atrapado hasta haber sellado la fístula para que la disminución de la presión intracraneal no incremente la entrada de aire. Nosotros consideramos que el drenaje por punción ventricular es una medida de urgencia que debe realizarse cuando la clínica lo demande ${ }^{8}$ y que la colocación de un taponamiento nasal anterior hasta el momento de la reparación definitiva del defecto dural puede ser útil en este contexto ${ }^{2}$.

\section{Bibliografía}

1. Açikbaş, S.C., Akyüz, M., Kazan, S., Tuncer, R.: Complications of closed continuous lumbar drainage of cerebrospinal fluid. Acta Neurochir 2002; 144: 475-480.

2. Altinörs, N., et al.: Tension pneumocephalus after transspenoidal surgery: case report. Neurosurgery 1988; 23: 516518.

3. Candrina, R., Galli, G., Bollati, A.: Subdural and intraventricular pneumocephalus after transsphenoidal operation. J Neurol Neurosurg Psychiatry 1988; 51: 1005-1006.

4. Haran, R.P., Chandy, M.S.: Symptomatic pneumocephalus after transsphenoidal surgery. Surg Neurol 1997; 48: 575-578.

5. Iplikcioglu, A.C., Bek, S., Bikmaz, K., Basocak, K.: Tension pneumocyst after transsphenoidal surgery for Rathke's cleft cyst: case report. Neurosurgery 2003; 52: 960963.

6. Kennedy, D.W., et al.: Transsphenoidal approach to the 
sella; the John Hopkins experience. Laryngoscope 1984; 94: 1066-1067.

7. Rao, G., Apfelbaum, R.I.: Symptomatic pneumocephalus occurring years after transsphenoidal surgery and radiation therapy for invasive pituitary tumor: a case report and review of the literature. Pituitary 2003; 6: 49-52.

8. Satyarthee, G.D., Mahapatra, A.K.: Tension pneumocephalus following transsphenoid surgery for pituitary adenoma - report of two cases. J Clin Neurosci 2003; 10: 495-497.

9. Sawka, A.M., Aniszewsski, J.P., Young, W.F., Nippoldt, T.B., Yanez, P., Ebersold, M.J.: Tension pneumocranium, a rare complication of transphenoidal surgery: Mayo Clinic experience 1976-1998. J Clin Endocrin Met 1999; 84: 47314734.

10. Sprague, A., Poulgrain, P.: Tension pneumocephalus: a case report and literature review. J Clin Neurosci 1999; 6: 418-424.

11. Thomas, D., Kendig, N.: Tension pneumocephalus:
Acute neurological deterioration associated with pleocytosis of the CSF. Clin Infect Dis 1993; 16: 703-705.

12. Walker, F.O., Vern, B.A.: The mechanism of pneumocephalus formation in patients with CSF fistulas. J Neurol Neurosurg Psychiatry 1986; 49: 203-205.

13. Yúceer, N., Çakíroğlu, K., Erdoğan, A., Gökalp, H.Z., Bağdatoğlu, C.: Tension pneumocephalus after transsphenoidal surgery: two case reports. Acta Neurochir 1995; 137: 58-61.

Ruiz-Juretschke, F.; Mateo-Sierra, O.; Iza-Vallejo, B.; Carrillo-Yagüe, R.: Neumoencéfalo intraventricular a tensión secundario a cirugía transesfenoidal: presentación de un caso y revisión de la literatura. Neurocirugía 2007; 18 : 134-137.

Correspondencia postal: Fernando Ruiz Juretschke. Servicio de Neurocirugía. Hospital General Universitario "Gregorio Marañón”. c/ Doctor Esquerdo 46 Madrid 28007 